
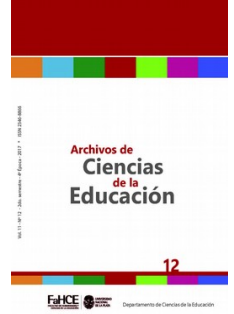

Archivos de Ciencias de la Educación, Vol. 11, n 12, diciembre 2017, e031. ISSN 2346-8866 Universidad Nacional de La Plata.

Facultad de Humanidades y Ciencias de la Educación.

Departamento de Ciencias de la Educación.

\title{
El aprendizaje significativo para fomentar el pensamiento crítico
}

\author{
The meaninful learning to promote the critical thinking
}

\section{Ricardo Chrobak}

Universidad Nacional del Comahue, Argentina | mecenster@gmail.com

\section{PALABRAS CLAVE}

Aprendizaje

Enseñanza

Pensamiento crítico

Creatividad

\section{KEYWORDS}

Learning

Teaching

Critical thinking

Creativity

\section{RESUMEN}

El artículo examina diversas conceptualizaciones y aspectos del aprendizaje significativo y su relación con el pensamiento crítico, modelos y herramientas tendientes al desarrollo de habilidades creativas para la resolución de problemas en ciencias exactas y naturales. Analiza experiencias educacionales de profesores universitarios y reflexiona sobre implicancias en la formación docente.

\section{ABSTRACT}

The article examines conceptualizations and aspects of meaningful learning and their relationship with critical thinking, models and tools for the development of creatives kills for problems olving in the natural and exacts ciences. It analyzes the educational experiences of university professors and reflects on the implications of teacher training. 


\section{Introducción}

Sabemos que nuestro país sufre de grandes postergaciones. Una larga tradición de enseñanza basada en teorías perimidas ha dado como resultado una muy pobre preparación de los estudiantes de hoy, que serán los ciudadanos del mañana. La situación se agrava cuando se trata de aprender asignaturas de las ciencias exactas y naturales, tan necesarias para lo que se denomina la alfabetización científico-tecnológica. Es cada vez menor el número de estudiantes universitarios que se matriculan o que se interesen por las carreras técnicas.

Considero que una propuesta adecuada para mejorar esta situación sería la aplicación del modelo de competencias, el cual generó nuevas formas de mediar los procesos de aprendizaje y evaluación en los estudiantes, al buscar que estuvieran en condiciones de identificar, interpretar, argumentar y resolver problemas pertinentes del contexto, siendo éstos los procesos mentales característicos del pensamiento crítico.

Se trata de lograr una socio-formación integral mediante el aprendizaje de las competencias necesarias para la autorrealización, la mejora del tejido social en lo local, lo nacional y la tierra patria, el desarrollo socioeconómico, el emprendimiento en las diversas áreas, la contribución al equilibrio y la sustentabilidad ambiental, y el fortalecimiento del arte, la cultura y la ciencia.

En este orden de ideas planteamos que, la educación tradicional — que parte de un docente que selecciona los contenidos de un programa, los organiza según su punto de vista y los explica, esperando que el educando aprenda esos contenidos y después los aplique - conlleva una lógica que ha sido rebasada por las demandas educativas actuales. "El paradigma de la 'educación centrada en las competencias’ promueve una lógica contraria: ahora es esencial enfrentarse a una tarea relevante (situada) que generará aprendizaje por la 'puesta en marcha' de todo el 'ser' implicado en su resolución"(Pimienta y Enríquez, 2009, p. 5). En definitiva, de lo que se trata es de "aprender a aprender y aprender a cambiar".

El concepto de aprendizaje significativo introducido por Ausubel (1968), resulta de particular aplicación para ser tomado como base en los diseños de los actuales métodos de enseñanza.

Otro concepto de aplicación es el de pensamiento crítico, que se caracteriza por estar constituido por habilidades cognitivas de alto nivel. Para lograrlo, se requiere que el estudiante domine principalmente los procesos de metacognición que constituyen una de las fortalezas potenciadas cuando se aplican las metodologías propias del logro de aprendizaje significativo, como clave para aprender a aprender y aprender a pensar.

Las relaciones entre el pensamiento crítico y el aprendizaje significativo son entonces evidentes. En este artículo se analizarán estas relaciones y cómo pueden aprovecharse sus interrelaciones para favorecer la formación de estudiantes y por ende, ciudadanos creativos. En efecto, las investigaciones sobre aprendizaje y memoria muestran que para desarrollar el pensamiento crítico son fundamentales la adquisición de competencias metacognitivas y la evaluación epistemológica, o sea aprender a pensar en lo que se piensa.

Pero, ¿qué es el pensamiento crítico? Se conoce así al proceso de pensamiento que involucra la evaluación analítica de una determinada situación. El pensar críticamente permite mejores desempeños y logros más importantes, a la vez que fortalece la creatividad. Todo ello optimiza las habilidades para resolver problemas y tomar decisiones.

Realmente, el uso del pensamiento crítico es una de las más importantes habilidades necesarias para el buen desempeño de las personas durante el siglo XXI. Esto se debe a que el pensamiento crítico involucra plantearse preguntas, analizar y evaluar -o emitir juicios de valor- basados en la información presentada. Todas estas características lo convierten en una de las habilidades primordiales a ser adquiridas por los 
estudiantes de todos los niveles educativos.

El pensamiento crítico involucra tres importantes etapas a seguir por el individuo cuando es enfrentado a una nueva información:

1. Análisis inicial

2. Evaluación de la información

3. Desarrollo de una posición personal sobre el problema basada en las propias lecturas, clases, tutoriales e investigaciones al respecto.

Además, para llegar a ser buenos pensadores críticos, debemos poder relacionar las teorías con las prácticas, hacer nuestras propias preguntas, buscar y utilizar nuevas evidencias, evaluar los argumentos, encontrar nexos entre los distintos argumentos y, finalmente, categorizar las líneas principales de pensamiento.

Estas últimas afirmaciones nos acercan al conocimiento y aplicación de las teorías de Ausubel, Novak y Gowin, hoy conocida como la teoría ANG, y a la aplicación de una de las herramientas metacognitivas desarrolladas en el marco de dicha teoría: los mapas conceptuales (Novak, J. D., and Gowin, D. B., 1981).

Una vez logrado el proceso de aplicación del pensamiento crítico, la mejor opción para internalizar lo aprendido, es proceder a la escritura crítica de los argumentos desarrollados y las soluciones adoptadas. En tal caso, se recomienda seguir la secuencia siguiente: a) categorizar los principales conceptos involucrados; b) establecer las interconexiones y relaciones de causas y efectos entre esos conceptos; c) realizar comparaciones y contrastes entre todos los conceptos centrales y puntualizar los problemas tratados; y, finalmente, d) proceder a la resolución de los problemas para sugerir las posibles soluciones obtenidas.

En este punto encontramos la aplicación de otra importante herramienta metacognitiva derivada de la teoría ANG, es decir la conocida como UVE de Gowin, también llamada epistemológica, heurística o UVE del conocimiento. "Esta herramienta se ha mostrado útil para facilitar la resolución de problemas” (Chrobak, R. 2010, p. 139).

Prácticamente, el desarrollo del pensamiento crítico estaría determinado por el cumplimiento de tres etapas, como se muestra a continuación:

\section{ACTITUD + CONOCIMIENTO + HABILIDADES DE PENSAMIENTO = PENSAMIENTO CRÍTICO}

Todas las definiciones asocian el pensamiento crítico a la racionalidad. Es el tipo de pensamiento que se caracteriza por manejar, dominar las ideas. Su principal función no es generar ideas sino revisarlas, evaluarlas y repasar qué es lo que se entiende, se procesa y se comunica mediante los otros tipos de pensamiento (verbal, matemático, lógico, etcétera). Por lo tanto, el pensador crítico es aquel capaz de pensar por sí mismo. El pensamiento crítico está formado tanto por habilidades como disposiciones, tal como lo han planteado autores como Ennis (2011); de conocimiento relevantes, como lo propone McPeck (1990); y competencias metacognitivas (Kuhn y Weinstock, 2002).

Finalmente, el pensador crítico debe evaluar las teorías y relacionarlas con las prácticas para llegar a una propuesta bien pensada y provocativa. Habiendo adquirido estas habilidades, los resultados lo ayudarán a entender los conocimientos importantes desarrollados en el siglo XXI, con posibilidades de participar en las decisiones importantes como ciudadano responsable y comprometido con el bienestar de toda la sociedad. 


\section{El aprendizaje significativo}

Trataré de describir brevemente, las ideas centrales y los principios del aprendizaje significativo y sus posibles aplicaciones para fortalecer el pensamiento crítico y, por lo tanto, lograr el mejoramiento de la enseñanza de las ciencias exactas y naturales.

En primer lugar, diremos que el aprendizaje puede tener múltiples grados de significatividad y que rara vez resulta $100 \%$ mecánico o $100 \%$ significativo sino que, en general, se ubica entre los extremos de un continuo que varía desde el puramente mecánico hasta el puramente significativo, tomando distintos grados de significatividad de acuerdo a cómo se fue adquiriendo el aprendizaje por parte del estudiante.

Ausubel sostiene que para que ocurra el aprendizaje significativo, es preciso que el alumno sea consciente de que él debe relacionar las nuevas ideas o informaciones que quiere incorporar a los aspectos relevantes de su estructura cognoscitiva. Esto no debe realizarse en forma arbitraria o “al pie de la letra" sino substancialmente.

Para que ello sea posible deben cumplirse ciertos requisitos, siendo los más importantes:

a. el material a ser aprendido debe ser potencialmente significativo, esto quiere decir, por ejemplo, que no se pueden enseñar significativamente listas de palabras sin sentido, las cuales, al no tener significado inherente, no podrán relacionarse con la estructura cognoscitiva,

b. el que está aprendiendo debe poseer conceptos y proposiciones relevantes en su estructura cognoscitiva que sean capaces de actuar como "base de anclaje" para las nuevas ideas a ser asimiladas,

c. el que está aprendiendo debe optar por relacionar intencionalmente el material potencialmente significativo, en forma no arbitraria y sustantiva, con la estructura cognoscitiva que ya posee.

Si alguna de estas condiciones falla, el aprendizaje significativo, y por lo tanto el pensamiento crítico, también se verá afectado.

\section{Los mapas conceptuales y el pensamiento crítico}

Los mapas conceptuales fueron desarrollados durante el transcurso de las investigaciones educativas realizadas por el Dr. J.D. Novak en la Cornell University (USA), donde se buscaba entender los cambios producidos en las estructuras de conocimiento de los estudiantes durante el aprendizaje de las ciencias.

Mediante investigaciones educativas sobre enseñanza de Física, ha sugerido en relación a enseñar creativamente que: los mapas conceptuales y la UVE del conocimiento son potenciadoras de la creatividad (Chrobak, R.et al., 2010).

Muchas veces nos preguntamos sobre el origen de los primeros conceptos que aprendimos. Ellos son adquiridos por los niños, desde el nacimiento hasta los tres años, mediante el reconocimiento de regularidades en el mundo que los rodea, comenzando a identificar también las etiquetas conceptuales o símbolos que representan a esas regularidades. Esta fenomenal habilidad es parte de la herencia evolutiva de todos los seres humanos.

Luego de los tres años, la adquisición de nuevos conceptos y proposiciones están fuertemente mediatizada por el lenguaje teniendo lugar, principalmente, a través del proceso de aprendizaje por recepción, en el cual los nuevos significados se obtienen formulando preguntas y clarificando las relaciones entre los conceptos y las proposiciones existentes y entre los conceptos y las proposiciones nuevos. Este proceso se ve fuertemente 
favorecido cuando se dispone de experiencias y soportes concretos. De allí la gran importancia de las actividades handson $\underline{\underline{1}}$ o experiencias de laboratorio en el aprendizaje de ciencias, especialmente para los estudiantes jóvenes, aunque esto también es cierto para los adultos.

\section{La UVE heurística de Gowin}

Otra de las herramientas metacognitivas desarrolladas en el marco de la teoría ANG, es la denominada UVE heurística ${ }^{2}$ o de Gowin, que pasaremos a describir a continuación.

Se llama heurística a la herramienta utilizada como ayuda para resolver un problema o comprender un procedimiento. La UVE heurística (o diagrama en "V") fue desarrollada en sus orígenes para ayudar a los estudiantes ya sus instructores a ver más claramente la naturaleza y finalidad de las prácticas de laboratorio en los cursos de Ciencias.

Es importante señalar que los elementos epistémicos están relacionados entre ellos y que, si estas relaciones no están bien establecidas en la estructura intelectual, el diagrama reflejará, posiblemente, alguna de las concepciones espontáneas. En tal caso, las elaboraciones futuras resultarán, probablemente, defectuosas. Esta propiedad del diagrama UVE de reflejar las concepciones espontáneas, es lo que lo hace particularmente útil para aplicarlo a la educación, ya que permite visualizar en forma simple las proposiciones que necesitan ser re-elaboradas.

Hemos ya señalado que para llegar a ser buenos pensadores críticos debemos poder relacionar las teorías con las prácticas, hacernos nuestras propias preguntas, buscar y utilizar nuevas evidencias, evaluar los argumentos, encontrar nexos entre los distintos argumentos y finalmente, categorizar las líneas de pensamiento principales. El uso de la UVE conduce al estudiante a realizar todas estas acciones de forma dinámica y creativa.

La hipótesis en relación a este punto es que la UVE potencia la creatividad de los individuos, por lo que, a continuación, describiremos someramente las características del ser creativo.

Aun reconociendo las numerosas definiciones de creatividad, es posible considerarla desde el punto de vista ausubeliano y en relación con el aprendizaje significativo, de donde surgen las condiciones mínimas que se requieren si se considera que la metacognición de cada sujeto sobre sus procesos creativos es una base teórica apropiada para elaborar cualquier método de formación de creatividad.

No es nuestra intención ahondar en la descripción de la creatividad por ser otras las motivaciones de este trabajo, pero sí podemos resumir estos criterios en un mapa conceptual como el que muestra la figura 1 (Novak, 1998, traducción del autor). 


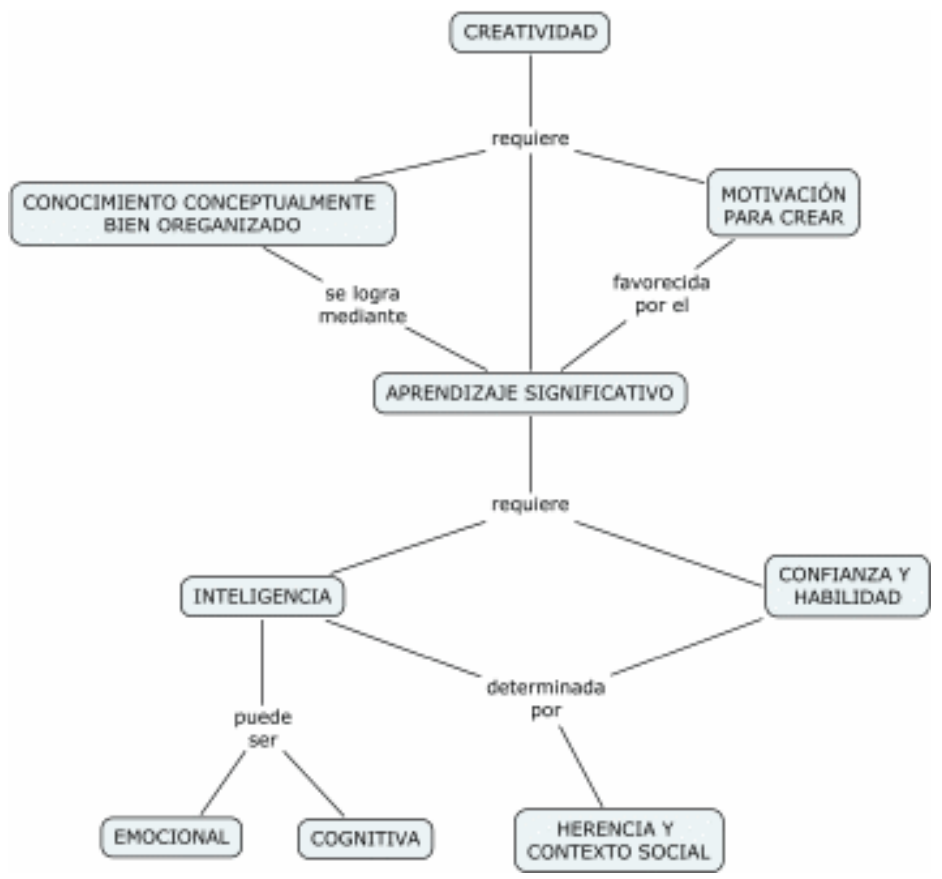

Figura 1. Conceptos centrales involucrados con la creatividad de los individuos

Cualquiera de nosotros posee capacidades creativas, pero no son muchos los que poseen la habilidad y el impulso emotivo para dar el salto que se necesita para producir adelantos en la ciencia, el arte, la música u otros campos del pensamiento humano. No obstante, los componentes creativos, pueden ser ampliamente mejorados desarrollando de modo significativo la capacidad y el deseo de aprender, en especial si logramos que el aprendizaje sea significativo.

Ser creativo involucra:

- Adquirir estructuras de conocimiento bien organizadas.

- Impulsar la búsqueda de conexiones entre diferentes dominios de conocimientos.

- Transformar estructuras de conocimiento conceptualmente opacas en estructuras conceptualmente transparentes.

- Integrar constructivamente el pensar, sentir y actuar a través de la experiencia.

Para lograr ciudadanos creativos ${ }^{3}$ como se requiere en el presente siglo, es importante que, en las prácticas educativas, tengamos en consideración que potenciar la creatividad ofrece grandes posibilidades. Sin embargo, la identificación del estudiante creativo es generalmente conflictiva y mal entendida por los profesores. Muchos creativos quedan marginados del sistema escolar por falta de una detección a tiempo y una ayuda adecuada.

El docente debe aplicar la práctica creativa en la docencia, sea como método de trabajo para o como propuesta de objetivo para el currículo. Una clase creativa es un foro para el pensamiento, un antídoto para el aburrimiento, una fuente de motivación para los estudiantes, un recurso garantizado ante las disrupciones y un "caldo de cultivo" para la excelencia. Debemos visualizar la creatividad como una manifestación de aquellos individuos que buscan altos niveles de adquisición de aprendizajes significativos. 
Cada faceta que caracteriza a la creatividad está relacionada con la búsqueda de integración de conceptos y proposiciones en una estructura cognitiva altamente organizada, como las que se obtienen con la buena construcción de mapas conceptuales. La recompensa afectiva que resulta de tal diferenciación y desarrollo cognitivo, cataliza el impulso creativo de cada individuo.

¿Cómo creemos que se pueden lograr estas aspiraciones?

- Buscando un verdadero cambio mental (sí, cambiar nuestra mentalidad) desde la opción convencional a la constructivista.

- Mediante los criterios emergentes de una teoría comprensible sobre el aprendizaje humano (aprendizaje significativo).

- Mediante la utilización de herramientas de enseñanza-aprendizaje que respondan a los criterios de la metacognición.

Todos tenemos una determinada idea respecto del significado del término "creatividad", sin embargo, la conceptualización desde el punto de vista científico, es un tema aún no del todo clarificado.

Al decir de Corbalán "estamos en una situación similar a la del descubrimiento de la electricidad: nadie sabía qué era aquello, pero se tenía una clara experiencia de su descarga. Probablemente hemos de tener mucha paciencia con el problema de la definición conceptual de la creatividad” (2015, p. 1).

Así, el desarrollo de un buen clima pedagógico de las clases debería caracterizarse por una serie de elementos que aportan a la formación de las competencias creativas, tales como: tener respeto por la individualidad del estudiante; centrar el proceso de formación en sus necesidades y posibilidades para elevar los niveles de motivación; tender a un proceso de enseñanza-aprendizaje que considere el ritmo de desarrollo de cada estudiante para lograr el pensamiento y el aprendizaje creativo; y, por último, propiciar la libertad y responsabilidad para experimentar, problematizar y discrepar desarrollando la independencia de acción, juicio e imaginación. Ninguna de estas características se cumplirá si no conseguimos que la docencia universitaria sirva para que los estudiantes se formulen sus propias preguntas.

A la luz de estas circunstancias, llama la atención la escasa presencia de la competencia creativa en el diseño de los nuevos planes de estudio de la Argentina. En especial, porque el conocido proyecto Tuning ${ }^{4}$ establece que la competencia creativa es una competencia genérica a desarrollar en todos los estudiantes universitarios, ya sea en las áreas de las ciencias naturales como en las sociales.

Una de las dimensiones características del ser creativo es la disposición natural y espontánea de manifestar curiosidad. La consecuencia de la curiosidad es el planteamiento constante de determinadas preguntas. Al respecto, Corbalán sostiene que:

El mundo se nos da resuelto, después lo problematizamos, le hacemos preguntas; más tarde las intentamos responder. Crear es todo ello; pero lo básico, el primer paso en la creatividad es elaborar preguntas. Si quisiéramos construir una máquina que piense, quizás deberíamos enseñarle antes a hacer preguntas. (Corbalán J.B. et al., 2015, p. 2).

Y justamente, aquí nos remitimos nuevamente a la UVE, cuyo principal objetivo inicial es hallar la pregunta central, motivo por el cual resulta conveniente recordar algunas citas referidas a la elaboración de preguntas.

A propósito de la importancia de la pregunta central, resultará de utilidad recordar distintos pensamientos que se popularizaron y hacen referencia a la relación entre plantearse preguntas y potenciar la creatividad: 
- La ciencia consiste en colgar preguntas a las certidumbres más logradas (Ortega y Gasset, 1936)

- El nacimiento de la ciencia moderna se remonta al momento en que, a las preguntas generales, las han sustituido las preguntas limitadas. En lugar de preguntar: ¿cómo ha sido creado el universo? o ¿cuál es la esencia de la vida? se ha preguntado: ¿cómo cae una piedra? o ¿cómo circula la sangre en el cuerpo? este cambio ha tenido un resultado sorprendente, mientras que las preguntas generales no recibían más que respuestas limitadas, las preguntas limitadas conducían a respuestas cada vez más generales (François Jacob, premio Nobel de Medicina, 1965)

- Cuando a Isidore Isaac Rabi ${ }^{5}$ (Descubridor de la resonancia magnética nuclear y premio Nobel de Física 1944), le preguntaron qué le había ayudado a ser Científico, respondió: Al salir de la escuela, todas las otras madres judías de Brooklyn, preguntaban a sus hijos: ¿qué habéis aprendido hoy en la escuela? En cambio, mi madre decía: ¿IZZY, te has planteado hoy alguna buena pregunta? (Christine Chin, 2004).

- La ciencia es por esencia pregunta, problematicidad. En cuanto pasamos del qué, el porqué de la consignación de los hechos hasta la develación de todos los fundamentos que los hacen inteligibles, empezamos a situarnos en la vertiente creadora (Heidegger, M., 1954).

- No debemos olvidar que lo que observamos no es la naturaleza misma, sino, la naturaleza determinada por la índole de nuestras preguntas (Heinsenberg, W.,1959). De esta última afirmación, resultaría que no es posible realizar la observación sin una teoría previa, necesaria para elaborar la pregunta, de allí se deriva también la validez del principio de la carga teórica de la observación.

Cada vez que el ser humano conquista un nuevo conocimiento parte de un interrogante. Lo que nos remite al primer elemento epistémico de la UVE que es la pregunta central, cuya importancia trataremos de ir analizando en función de cada uno de los componentes de la UVE.

El uso del diagrama UVE propuesto por Gowin permite imaginar, aproximadamente, una docena de los elementos epistémicos más relevantes que componen un cuerpo de conocimientos ocupando diferentes espacios de la estructura intelectual (Figura 2). La UVE ayuda a "desempaquetar" en forma imaginaria un determinado cuerpo de conocimientos y a analizar cada uno de sus distintos componentes epistemológicos, para luego reestructurarlos y reconstruirlos desde una nueva perspectiva, lo que permite obtener un cuerpo de conocimientos más amplio y evolucionado. 


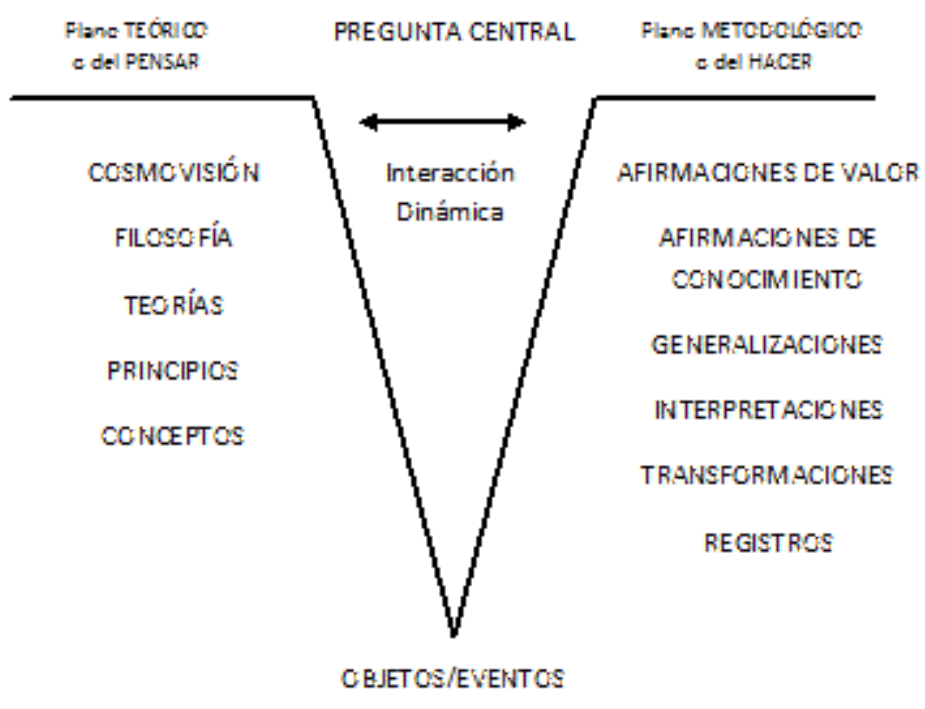

Figura 2. La UVE heurística como una representación de la estructura del conocimiento: una docena de elementos epistémicos. (Gowin, 1987)

Pregunta central: Una buena forma de delinear el contexto de trabajo es construir una pregunta central, esto es, una pregunta que especifique claramente el problema o asunto que la UVE ayudará a resolver. Por supuesto, la pregunta central o de enfoque, se debe referir a un objeto o evento. Las UVEs que se enfocan en eventos tienden a ser más ricas en explicaciones y las que se enfocan en objetos tienden a ser más descriptivas. Ambas ayudan a responder preguntas.

Cada vez que nos planteamos un problema, miramos el objeto o el acontecimiento a ser estudiado desde el marco conceptual de nuestro conocimiento previo. Es decir que el primer factor que influencia nuestra construcción de conocimientos, es justamente nuestra cosmovisión, o sea, nuestra "visión acerca del mundo que nos rodea". Los sujetos aceptan determinadas teorías que tratan de explicar "por qué" la naturaleza se comporta de la forma en que lo hace.

Los principios describen “cómo” se comporta la naturaleza, es decir que son menos elaborados que las teorías, a pesar de que muchas veces es difícil la distinción entre principio y teoría.

Según Ausubel, un concepto es el conjunto de regularidades percibidas en objetos o eventos que responden a una etiqueta (el nombre) y que tienen suficientes elementos en común como para permitirnos la comunicación.

El lado derecho de la UVE comienza con los registros. Los datos registrados a partir de las observaciones y mediciones constituyen los hechos.

El tratamiento de los registros nos lleva a las transformaciones. A menudo, analizar los registros en forma de gráficos conduce a los científicos a suponer que los gráficos son "maquinitas que ayudan a pensar". Lo mismo podemos mencionar sobre el gráfico UVE, al que podemos considerar como la "maquinita” que nos ayuda a empoderarnos para pensar críticamente.

Una vez que hemos acomodado y transformado toda la información proveniente de la observación y de las mediciones, pasamos finalmente a contestar su pregunta central, con una afirmación de conocimiento. Al no revestir el carácter de "hipótesis confirmada” las afirmaciones no constituyen la verdad última, por lo que pueden considerarse como verdades tentativas, que pueden dejar de serlo cuando nuevas evidencias científicas así lo indiquen, como sucedió con la teoría aristotélica heliocéntrica. 
Afirmaciones de valor: En esta etapa se formulan conclusiones que ya no están estrechamente relacionadas con el manejo de los datos, sino que son más bien estimaciones del que aprende vinculadas con el valor atribuido a las nuevas proposiciones halladas. Aquí es donde pueden comenzar los dramas. Recordemos que cuando Copérnico se dio cuenta de que era necesario modificar la cosmovisión y la filosofía que había sostenido, casi le cuesta la vida.

Las afirmaciones de valor son un paso hacia una intelectualización mayor del pensamiento complejo y crítico, que resalta la consideración de cuestiones éticas, morales y sentimentales. Con el uso de esta herramienta aparece clara la necesidad de aplicar el nuevo concepto de inteligencia emocional, tan desconsiderada en los pasos del método científico.

De esta manera, habremos podido identificar los componentes epistémicos intervinientes en la construcción de un nuevo cuerpo de conocimientos y no se nos escapará la profunda interrelación entre el lado izquierdo de la UVE (el lado del pensar o teórico) y el lado derecho de la UVE (el lado del hacer o metodológico), es decir, de qué manera todo nuevo conocimiento que incorporamos a nuestra estructura cognitiva modifica lo ya existente y cómo, a su vez, el conocimiento previo sirve de base a toda nueva construcción.

Comprendemos que, para trabajar con la UVE, no siempre resulta sencillo poder determinar la filosofía o la teoría con la que encararemos nuestras observaciones. El empleo de un mapa conceptual, que reemplace los distintos elementos epistemológicos, podrá igualmente explicitar el marco conceptual en que nos movemos. También podemos registrar en este sector las ideas previas de los estudiantes sobre un determinado fenómeno y permitirle luego confrontarlas con las afirmaciones de conocimiento y de valor que él mismo formule al final de la reconstrucción del conocimiento ocasionada por el estudio sistemático del mismo. Estaremos favoreciendo, entonces, el logro de los objetivos procedimentales y otorgando al estudiante una batería de recursos que harán posible la reconstrucción autónoma de nuevos conocimientos.

Evidentemente, para que el docente pueda transmitir entusiasmo a sus estudiantes en el uso de estas estrategias metodológicas, debe haberse apropiado con anterioridad de ellas y haberlas utilizado en sus tareas de planificación, evaluación, elaboración de materiales de lectura y estudio para su curso, entre otras.

Las herramientas metacognitivas han demostrado ser sólidamente eficaces, tanto en manos de los docentes, como en la de los estudiantes. Ciertamente, apropiarnos de estas estrategias requiere de un esfuerzo sostenido por parte de los docentes, ya que permanentemente debemos superar los "baches" existentes en nuestra propia estructura cognitiva. Pero, los resultados que pueden obtenerse en el aula al ver cómo, guiados por estos instrumentos poderosos, los estudiantes van construyendo aprendizajes significativos, superarán largamente las expectativas iniciales.

Así como escribir es siempre re-escribir, educar es re-educar. Es un continuo proceso de trabajar y re-trabajar, estructurar y re-estructurar las cualidades de la experiencia humana en interacción con la Naturaleza" (Gowin, D.B. 1981, p. 16).

\section{La UVE para fortalecer a docentes y estudiantes}

La UVE heurística nos muestra una forma de usar lo que ya sabemos y que constituyen los elementos epistemológicos sobre los que debemos pensar cuando intentamos realizar un nuevo aprendizaje. Al mismo tiempo, reconocemos que el conocimiento es construido por las personas, por lo que, evidentemente, habrá una estrecha relación entre el conocimiento que cada persona construye para sí misma y su aprendizaje.

Considerando que el aprendizaje está íntimamente enlazado al contexto de la educación, podemos analizar cómo las dos ramas principales de la UVE del conocimiento están relacionadas por actividades de preguntas $\mathrm{y}$ actividades de respuestas durante el aprendizaje humano, lo que la convierte en una herramienta que 
permite examinar el aprendizaje y es de vital importancia para la educación. El diagrama UVE en el contexto de la educación como una herramienta de aprendizaje, se muestra en la Figura 3.

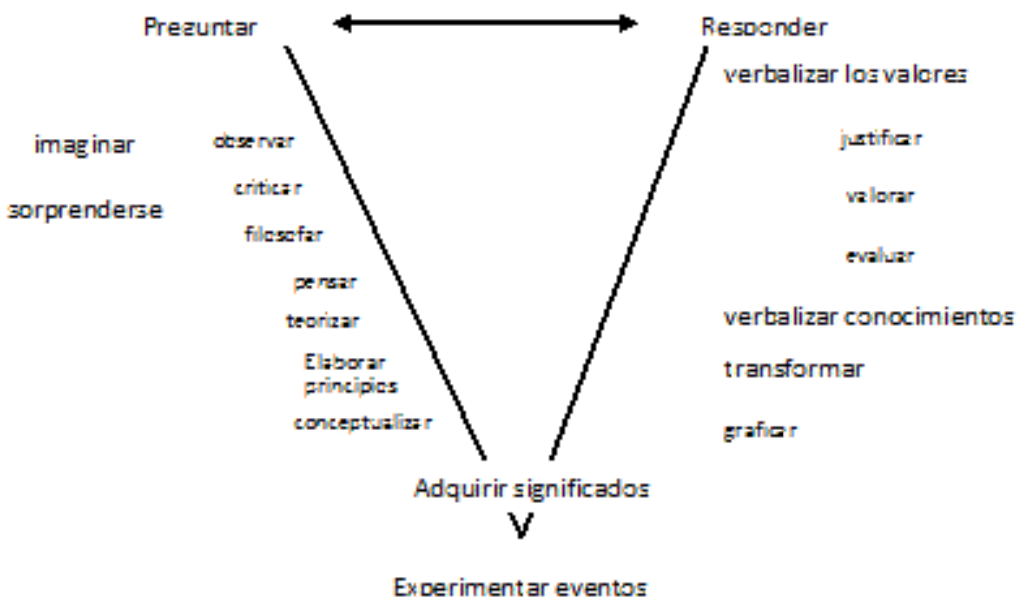

Figura 3. La UVE del Aprendizaje. (Gowin, 1987)

Por otra parte, Gowin destaca la importancia de relacionar, en una forma explícita y deliberada, el pensar, el actuar y el sentir. Podemos aprender a pensar a través del recorrido del lado izquierdo de la UVE (Lado del Pensar); guiar los procesos del actuar con el desarrollo del lado derecho de la UVE (Lado metodológico), mientras que los sentimientos son expresados ampliamente a través de la elaboración de afirmaciones de valor.

Numerosos trabajos de investigación han revelado muy buenos resultados cuando el docente busca que sus estudiantes transiten los pasos de la UVE del aprendizaje en la reconstrucción de conocimientos. Aprender es un proceso vital que tiende a la reorganización de la estructura cognitiva como actividad central.

En primer lugar, puede afirmarse que, en términos de esta postura constructivista, se considera el actuar como el comportamiento gobernado por el significado. Por lo tanto, el logro de aprendizajes significativos por parte de los estudiantes resultará en un cambio positivo de su forma de actuar. Aprender acerca del aprendizaje también lleva un cierto tiempo, siendo diferente para cada estudiante. El tiempo es un tirano de todo proceso de enseñanza- aprendizaje. No obstante, suele utilizarse para controlar directamente los esfuerzos, más que para verificar cuáles son los significados que dirigen esos esfuerzos.

Un segundo paso consiste en lograr que el estudiante llegue a ser competente en el análisis de la UVE. Puede ser a través del análisis de trabajos de otros, de ser posible, de gente que sea autoridad en ese tema. Es útil, asimismo, analizar trabajos de investigación, libros, manuales, artículos y las filosofías de la disciplina. El fortalecimiento de los estudiantes aparece cuando ellos llegan a comprender en qué medida la autoridad experta también es falible, ya que entre los expertos hay desacuerdos.

Un tercer momento se inicia cuando los estudiantes comienzan sus propias investigaciones. A medida que van completando sus trabajos, ellos se van dando cuenta de cuánto aprendieron por sí mismos. El trabajo del docente puede considerarse terminado cuando los trabajos de los estudiantes están bajo su propio control. Las entrevistas entre el docente y los estudiantes, grabadas o filmadas, son técnicas muy recomendables, que constituyen registros de nuevos eventos. Estos registros pueden ser estudiados por docentes y estudiantes para construir así una UVE de aprendizaje como estructura de conocimiento acerca del hecho educativo. "A medida que estos eventos vayan cambiando en el futuro, estas UVEs también cambiarán, fortaleciendo el proceso tanto de enseñar como de aprender” (Chrobak, R. et. al., 2010, 5). 


\section{Notas}

1 Aunque no resulta muy académica, la expresión más ilustrativa en nuestro idioma sería "con las manos en la masa”.

2 Heurístico: 1.- Del griego heuriskein, «hallar» «inventar». 2.- Técnica de la indagación y del descubrimiento. 3.- Búsqueda o investigación de documentos o fuentes históricas. 4.- En algunas ciencias, manera de buscar la solución de un problema mediante métodos no rigurosos, como por tanteo, reglas empíricas etc.

$\underline{3}$ El pensamiento creativo consiste en una nueva concepción de la creatividad, que busca que los individuos puedan cambiar el pensamiento espontáneo por el pensamiento crítico deliberado.

4 El proyecto Tuning es impulsado y coordinado por Universidades de distintos países europeos y latinoamericanos y tiene como propósito intercambiar experiencias para el desarrollo de la calidad educativa.

$\underline{5}$ Isidore Isaac Rabi (29 Julio 1898 - 11 enero 1988). Nacido en Ucrania (Galicia) nacionalizado estadounidense, fue reconocido con el premio Nobel de Física en 1944 por su descubrimiento de la resonancia magnética nuclear, ampliamente usada en medicina en el diagnóstico por imágenes. También participó en el desarrollo del Magnetrón de cavidad utilizado en el horno y el radar a microondas.

\section{Bibliografía}

Ausubel, D., Novak, J. D., \& Hanesian, H. (1978). Educational Psychology, a cognitive view. 2nd Edition. New York: Holt, Rinehart and Wiston.

Corbalán Berná, F. J. et al. (2015). CREA. Inteligencia Creativa. Una medida cognitiva de la Creatividad. Madrid: TEA Ediciones.

Chrobak, R. (2010). Volver a Aprender, el derecho a Enseñar. Neuquén: Editorial EDUCO, U.N.Co.

Chrobak, R. et. al. (2010). Enseñar creativamente: los mapas conceptuales y la uve del conocimiento ¿pueden fomentar la creatividad? Memorias. CMC 2010, Vol. 1, 222-229. Viña del Mar, Chile

Chrobak, R., Prieto, A. B. (2010). La herramienta UVE del conocimiento para favorecer la creatividad de docentes y estudiantes. En Anales de Psicología, 26(2), 197-418.

Ennis \& Halone (2011). Pensamiento crítico en el aula. Recuperado de: educacion.to.uclm.es/pdf/revistaDI/3_22_2012.pdf

Kuhn, D., Weinstock, M. (2002). What is epistemological thinking and why does it matter? Department of Psychology, Teachers College, Columbia University, Box 119, NY 10027, USA.

Gowin, D.B. (1981). Educating. Ithaca, New York: Cornell University Press.

McPeck, J. E. (1990). Teaching Critical Thinking: Dialogue and Dialectic. Front Cover. J. E. McPeck. Routledge, Chapman \& Hall, Incorporated.

Novak, J. D. (1998). Learning, Creating, and Using Knowledge. Concept Maps as Facilitative Tools in Schools and Corporations. Mahwah, New Jersey, London: Lawrence Erlbaum associates, publishers.

Novak, J. D., \& Gowin, D. B. (1981). Concept Mapping and Other innovative Strategies. Unpublished manuscript, Cornell University.

Pimienta, J. y Enríquez, A. (2009). Educación basada en competencias. Guía para la aplicación del enfoque. México: Pearson. 